\title{
Литература
}

1. Лаптинский В.Н. Многоточечная задача управления с квазиразделенными краевыми условиями // Материалы, оборудование и ресурсосберегающие технологии: материалы междунар. науч.техн.конф., Могилев, 16-17 апреля 2015 г. Могилев: Белорус.-Рос. ун-т, 2015. С. 325.

2. Лаптинский В.Н. Конструктивный анализ управляемых колебателъных систем. Мн.: ИМ НАН Беларуси, 1998.

3. Бондарев А.Н Многоточечная краевая задача для уравнения Ляпунова в случае сильного вырождения краевых условий // Дифференц. уравнения. 2011. Т. 47, № 6. С. 776-784.

4. Демидович Б. П. Лекиии по математической теории устойчивости. М.: Наука, 1967.

\section{КРИТЕРИИ МОДАЛЬНОЙ УПРАВЛЯЕМОСТИ ЛИНЕЙНЫХ СИСТЕМ НЕЙТРАЛЬНОГО ТИПА}

\author{
А.В. Метельский ${ }^{1}$, В.Е. Хартовский ${ }^{2}$, \\ ${ }^{1}$ Белорусский национальный технический университет, Минск, Беларусь \\ ametelski@bntu.by \\ ${ }^{2}$ Гродненский государственный университет им. Я. Купалы, Гродно, Беларусь \\ hartovskij@grsu.by
}

Пусть задана линейная автономная дифференциально-разностная система нейтрального типа с соизмеримыми запаздываниями

$$
\dot{x}(t)-\sum_{i=1}^{m} D_{i} \dot{x}(t-i h)=\sum_{i=0}^{m}\left(A_{i} x(t-i h)+B_{i} u(t-i h)\right), \quad t>0,
$$

где $D_{i} \in \mathbb{R}^{n \times n}, A_{i} \in \mathbb{R}^{n \times n}, B_{i} \in \mathbb{R}^{n \times r}, x$ - вектор решения, $u$ - вектор управления, $h=$ const $>0$. Определим полиномиальные матрицы $D(\lambda)=\sum_{i=1}^{m} \lambda^{i} D_{i}, A(\lambda)=\sum_{i=0}^{m} \lambda^{i} A_{i}$, $B(\lambda)=\sum_{i=0}^{m} \lambda^{i} B_{i}$ и перепишем исходную систему в операторном виде

$$
\left(E_{n}-D(\lambda)\right) \dot{x}(t)=A(\lambda) x(t)+B(\lambda) u(t), \quad t>0,
$$

где $\lambda$ - оператор сдвига, определяемый правилом $\lambda^{k} f(t)=f(t-k h)$ (для произвольной функции $f), \quad E_{i} \in \mathbb{R}^{i \times i}$ - единичная матрица.

Квазиполином вида $\Delta\left(p, e^{-p h}\right)=\sum_{i=0}^{\phi} p^{i} \Delta_{i}\left(e^{-p h}\right)$, где $\Delta_{i}(\lambda)$ - некоторые полиномы, причем $\Delta_{n}(0)=1$, будем называть квазиполиномом нейтрального типа степени $\phi$. В частности, характеристический квазиполином $\left|W\left(p, e^{-p h}\right)\right|$ разомкнутой $(u \equiv 0)$ системы (1), где $W\left(p, e^{-p h}\right)=p\left(E_{n}-D\left(e^{-p h}\right)\right)-A\left(e^{-p h}\right)$ - характеристическая матрица системы (1), $|\cdot|$ - определитель матрицы, является в общем случае квазиполиномом нейтрального типа степени $n$.

Рассмотрим два класса регуляторов с обратной связью по состоянию, не выводящих замкнутую систему из множества систем нейтрального типа:

1) класс регуляторов постоянной структуры, имеющих вид

$$
\begin{gathered}
u(t)=\widehat{G}_{1}(\lambda) X(t)+G_{1}(\lambda) \dot{X}(t-h)+\sum_{i=0}^{m_{1}} \int_{0}^{h} R_{1, i}(s) \lambda^{i} X(t-s) d s, \\
\dot{x}^{*}(t)=\widehat{G}_{2}(\lambda) X(t)+G_{2}(\lambda) \dot{X}(t-h)+\sum_{i=0}^{m_{1}} \int_{0}^{h} R_{2, i}(s) \lambda^{i} X(t-s) d s, \quad t>0 ;
\end{gathered}
$$


2) класс регуляторов переменной структуры, имеющих вид

$$
\begin{gathered}
u(t)=\widehat{C}_{1}(\lambda) X(t)+C_{1}(\lambda) \dot{X}(t-h)+\sum_{i=0}^{m_{2}} \int_{0}^{h} F_{1, i}(s) \lambda^{i} X(t-s) d s+T \psi(t), \\
\psi(t)=S \psi(t-h)+\widehat{C}_{2}(\lambda) X(t)+C_{2}(\lambda) \dot{X}(t-h)+\sum_{i=0}^{m_{2}} \int_{0}^{h} F_{2, i}(s) \lambda^{i} X(t-s) d s, \\
\dot{x}^{*}(t)=\widehat{C}_{3}(\lambda) X(t)+C_{3}(\lambda) \dot{X}(t-h)+\sum_{i=0}^{m_{2}} \int_{0}^{h} F_{3, i}(s) \lambda^{i} X(t-s) d s, \quad t>0,
\end{gathered}
$$

где $x^{*}, \psi$ - вспомогательные переменные, $X=\operatorname{col}\left[x, x^{*}\right], \widehat{G}_{i}(\lambda), G_{i}(\lambda), i=1,2, \widehat{C}_{i}(\lambda)$, $C_{i}(\lambda) i=\overline{1,3}$, - полиномиальные матрицы, $T, S$ - постоянные матрицы,

$$
\begin{gathered}
R_{j, i}(s)=\sum_{k=0}^{m_{1}^{*}} e^{\alpha_{1, k, i, j} s}\left(\cos \left(\beta_{1, k, i, j} s\right) R_{j, i, 1, k}(s)+\sin \left(\beta_{1, k, i, j} s\right) R_{j, i, 2, k}(s)\right), \quad j=1,2, \quad i=\overline{1, m_{1}}, \\
F_{j, i}(s)=\sum_{k=0}^{m_{2}^{*}} e^{\alpha_{2, k, i, j} s}\left(\cos \left(\beta_{2, k, i, j} s\right) F_{j, i, 1, k}(s)+\sin \left(\beta_{2, k, i, j} s\right) F_{j, i, 2, k}(s)\right), \quad j=1,3, \quad i=\overline{1, m_{2}}, \\
\left(\alpha_{e, k, i, j}, \quad \beta_{e, k, i, j} \in \mathbb{R}, \quad R_{j, i, e, k}(s), \quad F_{j, i, e, k}(s)-\text { полиномиальные матрицы }\right) .
\end{gathered}
$$

Если подставить в систему (1) управление $u$, определяемое соотношением $(2)$, и добавить уравнение (3), то при $t>m h$ получим замкнутую систему нейтрального типа (1)-(3). Уточним, что будем понимать под замкнутой системой $(1),(4)-(6)$. При любой заданной функции $\psi(t), t \leqslant 0$, на каждом полуинтервале $(k h,(k+1) h], k=0,1, \ldots$, функция $\psi(t)$ выражается через величины $X(\tau), \dot{X}(\tau-h), \tau \leqslant t$, согласно разностному уравнению (5). После ее подстановки в (4) получаем функцию $u(t), t \in(k h,(k+1) h]$, зависящую от $X(\tau), \dot{X}(\tau-h)$, $\tau \leqslant t$, которая в совокупности с уравнением (6) определяет регулятор на полуинтервале $(k h,(k+1) h]$. Замыкая им систему $(1)$, на полуинтервале $(k h,(k+1) h], k=m, m+1, \ldots$, получим линейную дифференциально-разностную систему нейтрального типа с соизмеримыми запаздываниями. На следующем полуинтервале описанный процесс повторяется. Определенную таким образом при $t>m h$ систему будем называть системой $(1)$, замкнутой регулятором (4)-(6), а под ее решением будем понимать функцию $X$. Из сказанного также следует, что на каждом полуинтервале $(k h,(k+1) h], k=0,1, \ldots$, регулятор $(4)-(6)$ меняет свою структуру как функция состояния. Параметры регулятора (4)-(6) выбираются таким образом, чтобы, невзирая на переменную структуру регулятора (4)-(6), замкнутая системы (1), (4)-(6) при $t>m h$ стала однородной линейной автономной системой нейтрального типа.

Обозначим $W_{1}\left(p, e^{-p h}\right)$ и $W_{2}\left(p, e^{-p h}\right)$ - характеристические матрицы замкнутых систем (1)-(3) и (1), (4)-(6) соответственно.

Определение 1. Систему (1) назовем модально управляемой в классе регуляторов постоянной (переменной) структуры, если для любого наперед заданного квазиполинома $d\left(p, e^{-p h}\right)$ нейтрального типа степени $n^{*}, n^{*} \geqslant n_{1}, \quad\left(n_{1} \in \mathbb{N}\right.$ - фиксированное число) существует регулятор (2), (3) ((4)-(6)) такой, что $\left|W_{1}\left(p, e^{-p h}\right)\right|=d\left(p, e^{-p h}\right) \quad\left(\left|W_{2}\left(p, e^{-p h}\right)\right|=d\left(p, e^{-p h}\right)\right)$.

В докладе приводятся критерии модальной управляемости системы (1) в классах регуляторов (2), (3) и (4)-(6), а также конструктивные методы их построения. Развитые в исследовании методы построения регуляторов (2), (3) и (4)-(6) базируются на элементарных преобразованиях полиномиальных матриц и не требует знания полного спектра исходной системы (1). 